\title{
In situ TEM Raman spectroscopy and laser-based materials modification
}

\author{
F. I. Allen ${ }^{\mathrm{a}, \mathrm{b}}$, E. Kim ${ }^{\mathrm{c}}$, N. C. Andresen ${ }^{\mathrm{d}}$, C. P. Grigoropoulos ${ }^{\mathrm{c}}$, A. M. Minor ${ }^{\mathrm{a}, \mathrm{b}}$ \\ ${ }^{a}$ Department of Materials Science and Engineering, University of California, Berkeley, California 94720, USA \\ ${ }^{b}$ National Center for Electron Microscopy, Molecular Foundry, Lawrence Berkeley National Laboratory, Berkeley, California 94720, USA \\ ${ }^{c}$ Department of Mechanical Engineering, University of California, Berkeley, California 94720, USA \\ ${ }^{d}$ Engineering Division, Lawrence Berkeley National Laboratory, Berkeley, California 94720, USA
}

\begin{abstract}
We present a modular assembly that enables both in situ Raman spectroscopy and laser-based materials processing to be performed in a transmission electron microscope. The system comprises a lensed Raman probe mounted inside the microscope column in the specimen plane and a custom specimen holder with a vacuum feedthrough for a tapered optical fiber. The Raman probe incorporates both excitation and collection optics, and localized laser processing is performed using pulsed laser light delivered to the specimen via the tapered optical fiber. Precise positioning of the fiber is achieved using a nanomanipulation stage in combination with simultaneous electron-beam imaging of the tip-to-sample distance. Materials modification is monitored in real time by transmission electron microscopy. First results obtained using the assembly are presented for in situ pulsed laser ablation of MoS $\mathrm{M}_{2}$ combined with Raman spectroscopy, complimented by electron-beam diffraction and electron energy-loss spectroscopy.
\end{abstract}

Keywords: in situ TEM, Raman spectroscopy, pulsed laser ablation, $\mathrm{MoS}_{2}$

\section{Introduction}

Raman spectroscopy is a versatile tool for the characterization of molecules and condensed matter, probing inelastic scattering interactions of photons with vibrational, rotational and other low-frequency modes. In materials science, Raman spectroscopy is thus used to probe plasmons, phonons, and magnons, providing information on chemical composition, temperature, stress/strain, and magnetization[1]. The laser beam used for the excitation can be scanned across a sample to generate Raman spectra pixel by pixel. Through integration onto a microscope platform, these Raman images can then be correlated directly with the information yielded by various other imaging techniques. For example, integrated assemblies for confocal microscopy[2], scanning electron microscopy (SEM)[3], and atomic force microscopy (AFM)[4] have been developed, thereby combining Raman spectroscopy with different flavors of surface imaging at various spatial resolutions. The focus of the work presented here is on a new approach for combining Raman spectroscopy with transmission electron microscopy (TEM), paving the way to rich datasets in which Raman signals can be correlated directly with structural features and chemical compositions in small volumes imaged at the nanoscale.

A major challenge in Raman spectroscopy is the limited signal strength, since the cross sections for Raman scattering are inherently low. Therefore, efficient methods for coupling light to and from the sample are required. If the in situ experiment is to take place in vacuum, as is the case with SEM and TEM, then

Email addresses: fiallen@lbl.gov (F. I. Allen), aminor@lbl.gov (A. M. Minor) a low-loss means to couple optics to the sample in the vacuum chamber of the microscope is needed. In addition, in the case of TEM, the Raman assembly must be designed to meet the stringent height requirements imposed by the pole-piece gap in the objective lens, which is at most $\sim 10 \mathrm{~mm}$. In the in situ TEM Raman approaches developed thus far[5, 6], optical access is achieved through a viewport installed on the microscope column. A parabolic mirror mounted near the specimen is then used to focus the incoming light and to collect the outgoing optical signals that pass back through the viewport to the spectrometer. In situ TEM Raman spectra have been acquired for diamond[5], silicon[6], and carbon nanotubes[6], employing optical illumination in the far field with spot sizes of several microns. Picher et al., whose setup is integrated into a microscope optimized for environmental TEM, have also demonstrated the measurement of sample temperature via the downshift of Raman peaks[6].

Here we introduce a novel modular system for in situ TEM Raman comprising a lensed Raman probe with optics for excitation and collection, and a nanomanipulation sample holder with a vacuum feedthrough for a tapered optical fiber. The assembly is installed on an electron microscope equipped with an energy filter, hence enabling electron energy-loss spectroscopy (EELS) to compliment the Raman spectra. Since the Raman probe is installed inside the microscope column and collects optical signals from the sample directly, mirrors and an optical viewport are not required. The direct coupling of an optical fiber to the sample chamber via the sample holder follows a method developed previously and enables localized laser illumination (pulsed or continuous wave) through close approach of a tapered fiber to the specimen for nanoscale in situ investi- 


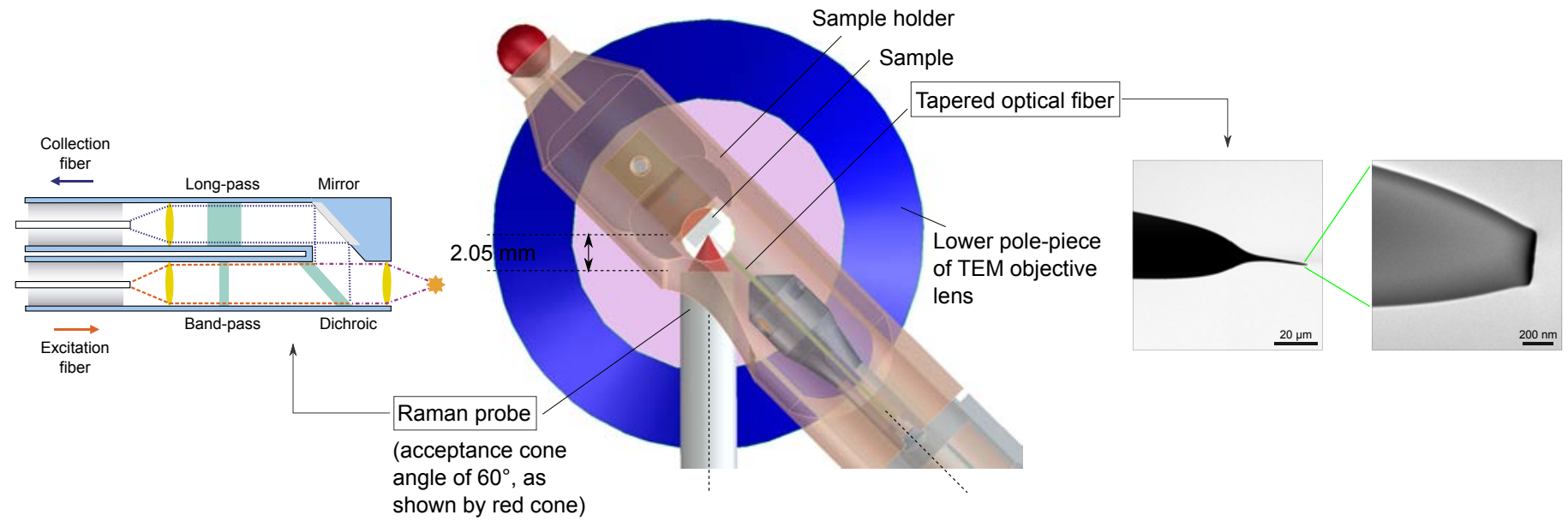

Figure 1: Layout of in situ Raman assembly in sample chamber of TEM (middle), schematic of InPhotonics RamanProbe ${ }^{\mathrm{TM}}$ (left), and TEM images of tapered optical fiber (right).

gations of laser-induced materials interactions[7].

\section{Experimental}

The layout of the in situ TEM Raman assembly highlighting the main components, i.e. a Raman probe module mounted on the sample chamber and a tapered optical fiber coupled to the sample holder, is shown in Figure 1.

The Raman probe is a customized lensed probe from InPhotonics that incorporates optics for excitation as well as collection. The probe is mounted on an $\mathrm{x}-\mathrm{y}-\mathrm{z}$ micrometer-based translation stage on a vacuum port in the specimen plane at an angle of $45^{\circ}$ to the sample port. Optical access to the sample is achieved by translating the end of the probe through an opening in the side of the customized sample holder. The probe tip is moved to a distance of $\sim 2 \mathrm{~mm}$ from the sample, at which point the focal point of the lens lands on the sample and the signal acceptance cone (shaded in red in the schematic) subtends an angle of $60^{\circ}$.

A schematic showing the two optical pathways in the Raman probe is shown on the left hand side of Figure 1. A $532 \mathrm{~nm}$ continuous-wave DPSS laser is coupled to a $50 \mu \mathrm{m}$ optical fiber and used to deliver up to $20 \mathrm{~mW}$ to the specimen. In the excitation pathway of the probe a band-pass filter attenuates the Raman bands from silica that are generated by photon scattering in the optical fiber. The laser light is focused onto the specimen via the lens at the end of the probe giving a spot size of $\sim 2 \mu \mathrm{m}$, calculated using $N A=0.2 \simeq \lambda_{0} / \pi \omega_{0}$, where $N A$ is the numerical aperture of the probe $(0.2), \lambda_{0}$ is $532 \mathrm{~nm}$, and $2 \omega_{0}$ is the spot size (a factor of $20 \%$ increase in size due to spherical aberrations is assumed). Scattered light collected by the probe is reflected into the collection optical pathway via a dichroic mirror. A long-pass filter attenuates Rayleigh-scattered light (i.e. elastically scattered photons; the dominant signal) and a $62.5 \mu \mathrm{m}$ optical fiber then delivers the inelastically scattered photons to the Raman spectrometer. During alignment, the intensity of a strong Raman peak from the sample, e.g. the Si-Si stretch vibration at $520 \mathrm{~cm}^{-1}$, is monitored and the $\mathrm{x}-\mathrm{y}-\mathrm{z}$ probe translation stage is used to adjust the probe position to maximize signal collection. The Raman spectrometer is a Renishaw InVia spectrometer that has been decoupled from its standard confocal microscope in order to couple it to the TEM instead.

In addition to the opening in the side of the sample holder that allows access of the Raman probe, the holder is also customized with a nanomanipulation stage and vacuum feedthrough for the tapered optical fiber of our in situ setup, following a design introduced previously[7]. Using a tapered fiber (shown on the right of Figure 1) and moving the tip close to the specimen using the nanomanipulation stage, highly localized laser illumination of the specimen is achieved at precisely controlled locations. Tapering of the fiber (core diameter $3 \mu \mathrm{m}$ ) is performed using a laser-based micropipette puller (P-2000 Sutter Instrument) achieving fiber-tip aperture sizes of typically 200$400 \mathrm{~nm}$.

Alignment of the fiber tip to the region of interest on the sam-
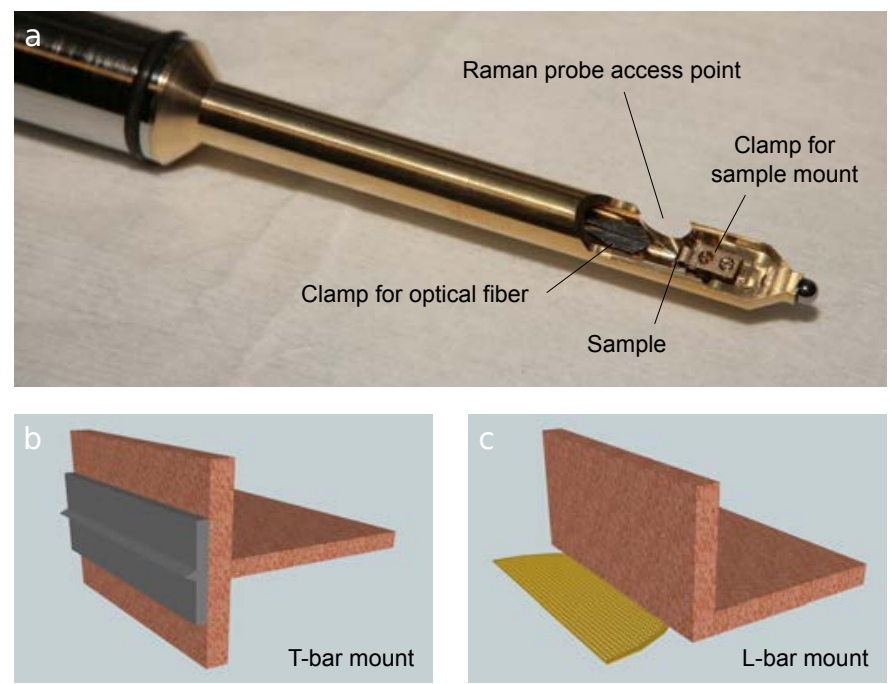

Figure 2: (a) Photo showing tip of sample holder. Schematics showing (b) T-bar and (c) L-bar sample mount geometries. 

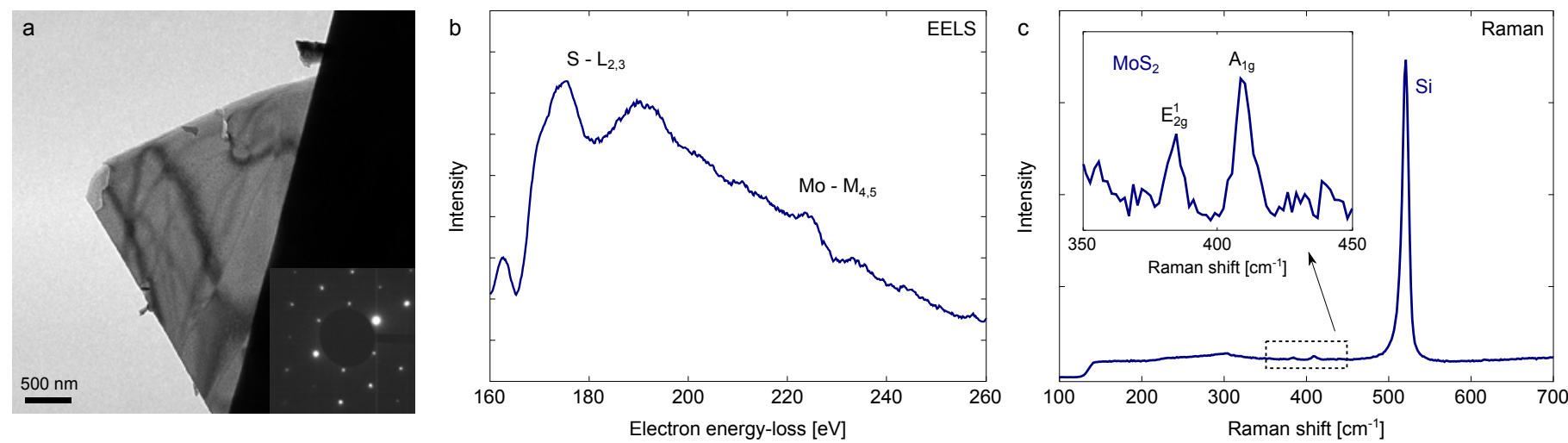

Figure 3: (a) Bright-field TEM of $\mathrm{MoS}_{2}$ flake on Si substrate. (b) TEM selected area diffraction of MoS 2 flake. (c) Corresponding EELS and (d) in situ Raman spectra.

ple is accomplished by viewing the tip-to-sample distance using the CCD camera of the TEM and adjusting the tip position first using the coarse positioning of an $x-y-z$ micrometer-based translation stage installed at the barrel-end of the holder and then using the fine positioning enabled by ceramic piezos for $\mathrm{x}$ $y-z$ motion installed in the holder shaft. Since the tip-to-sample distance is monitored in real-time by TEM imaging, positioning with nanometer precision is enabled. N.B. the height of the fiber tip, or z-position, is aligned to the height of the specimen using the objective wobbler function of the TEM, i.e. when both the sample and tip are at minimum focus (zero objective wobble), then they are at the same height. Two lasers are coupled to the tapered fiber that can be used interchangeably. One is a $532 \mathrm{~nm}$ continuous-wave DPSS laser and the other is a $532 \mathrm{~nm}$ pulsed Nd:YAG laser (3-4 ns pulses, $50 \mathrm{~mJ} /$ pulse, $10 \mathrm{~Hz}$ ) that is used for high power-density material processing.

Figure $2 \mathrm{a}$ shows a photograph of the tip of the sample holder. Two types of sample mount are used, as shown in the schematics of Figures $2 b$ and c. One is a T-bar mount, which is used for silicon wedge substrates (microfabricated using an anisotropic wet etch) onto which various samples can be deposited, e.g. nanoparticles or thin films. The other is an L-bar mount, which is used for TEM half-grid substrates. Using these geometries, the sample can be viewed in transmission using the TEM and can also be accessed from the side with the Raman probe and/or the tapered optical fiber. The substrates are fixed to the appropriate mount using Crystalbond ${ }^{\mathrm{TM}}$ adhesive and the mount is then installed into the sample holder using the metal clamp near the tip end.

The in situ assembly described is installed on a Zeiss Libra 200 TEM, which incorporates an in-column energy filter. Hence in addition to standard TEM imaging and TEM diffraction, elemental and chemical analysis complimentary to the Raman spectra can be obtained by electron energy-loss spectroscopy (EELS)[8].

\section{Results}

We demonstrate the versatility of the in situ TEM Raman setup by presenting a set of results obtained for an experiment on mechanically exfoliated thin-film $\mathrm{MoS}_{2}$. As shown in Figure 3, a range of characterization techniques is possible on the same sample inside the TEM using the experimental setup described. A bright-field TEM image of an $\mathrm{MoS}_{2}$ thin-film flake on an Si substrate is shown in Figure $3 \mathrm{a}$ with the corresponding TEM diffraction pattern inset. Core-loss EELS results are given Figure 3b, and the corresponding in situ unprocessed Raman spectrum in Figure 3c. The diffraction pattern demonstrates the single-crystal nature of the thin film and the EELS results show the presence of $\mathrm{S}$ and Mo by the characteristic $\mathrm{L}_{2.3}$ - and $\mathrm{M}_{4,5}$-shell ionization edges at $\sim 175$ and $225 \mathrm{eV}$, respectively. The Raman spectrum confirms the chemical composition of the sample, capturing the in-plane $\mathrm{E}_{2 g}^{1}$ and out-of-plane $\mathrm{A}_{1 g}$ vibration modes of $\mathrm{MoS}_{2}$ and the intense Si vibration mode of the wedge substrate at $520 \mathrm{~cm}^{-1}$, characteristic of unstrained crystalline Si. The Raman shifts of the $\mathrm{MoS}_{2} \mathrm{E}_{2 g}^{1}$ and $\mathrm{A}_{1 g}$ peaks are measured at 382 and $407 \mathrm{~cm}^{-1}$, respectively, in good agreement with literature values[9].

In a subsequent experiment on a similar $\mathrm{MoS}_{2}$ flake, the tapered optical fiber of our in situ assembly was used to deliver highly localized intense laser pulses to the specimen causing the removal of material by laser ablation. Figures $4 a$ and $b$ show TEM images of an $\mathrm{MoS}_{2}$ flake recorded before and after a series of pulses were applied using the Nd:YAG laser. The energy output of the laser was adjusted until visible damage to the $\mathrm{MoS}_{2}$ flake was observed, requiring on the order of $1 \mathrm{~J} \mathrm{~cm}^{-2}$. Figure $4 \mathrm{c}$ shows a single frame taken from a movie of TEM images that was recorded during the ablation process. Material was ablated from the flake starting from the left side (i.e. closest to the fiber tip) moving to the right. A brighter (thinner) region was observed during the ablation, shown outlined by a red dashed box in Figure 4c. Hence the indication is that the ablation process involved a stepwise thinning of the freestanding film, similar to the laser-induced thinning of supported $\mathrm{MoS}_{2}$ flakes reported elsewhere[10]. Upon further ablation, the thinned region was entirely removed. Closer inspection of the sample after the pulse processing reveals approximately spherical crystalline particles with a narrow size distribution of 20$30 \mathrm{~nm}$ that formed near the edge of the ablated region. A group of these nanoparticles is outlined in Figure $4 \mathrm{~b}$ (red dashed box) 

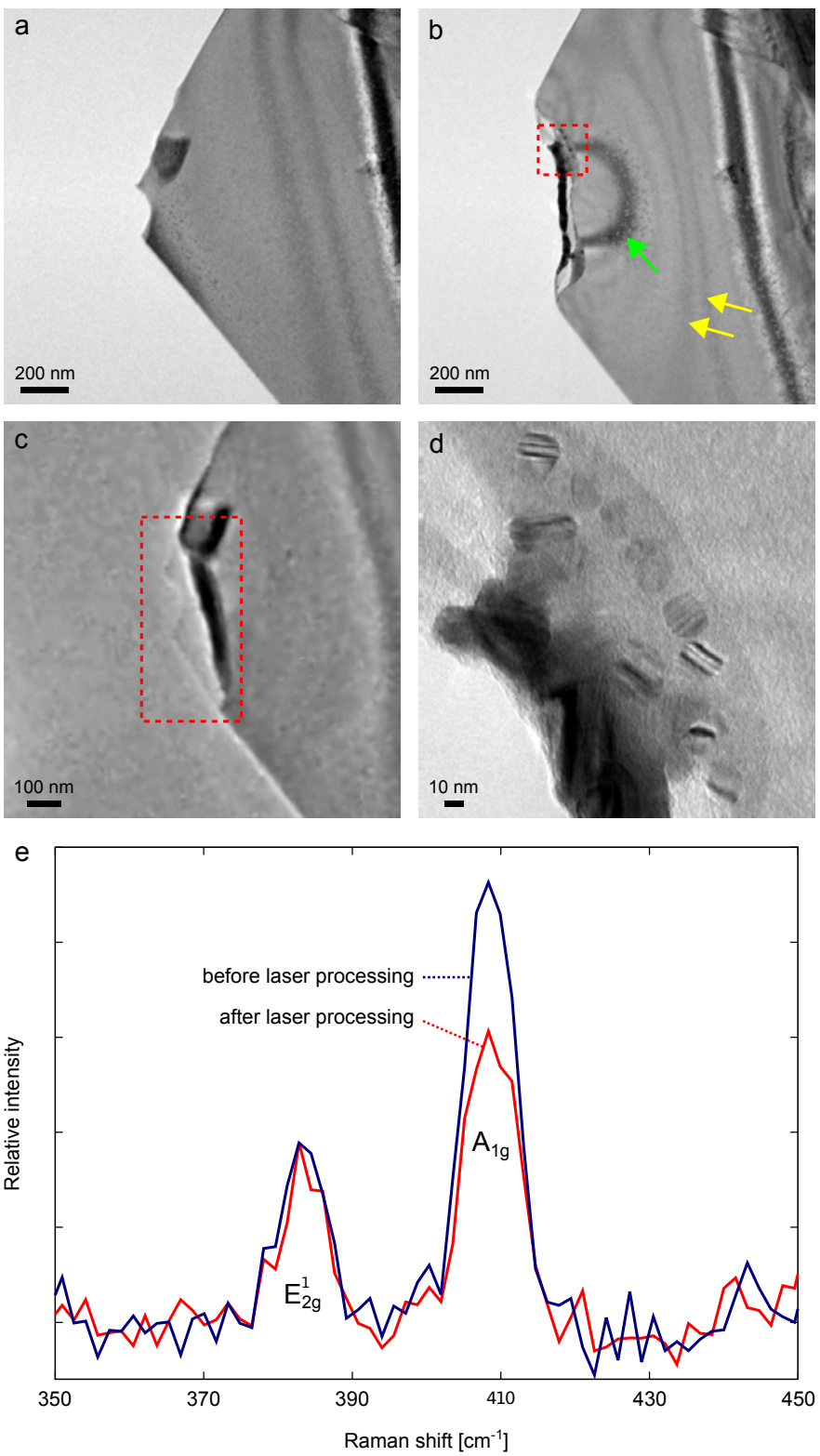

Figure 4: Bright-field TEM of $\mathrm{MoS}_{2}$ flake (a) before and (b) after pulsed laser ablation. (c) Bright-field TEM snapshot from movie of the $\mathrm{MoS}_{2}$ flake acquired during laser processing. (d) Higher magnification view of (b) showing spherical crystallites formed as a result of the pulsed laser processing. (e) Raman spectra of $\mathrm{MoS}_{2}$ flake acquired before (blue) and after (red) laser processing, normalized to the $\mathrm{E}_{2 g}^{1}$ peak and background-subtracted.

and shown at higher magnification in Figure 4d. The formation of nanoparticles by pulsed laser ablation of materials in vacuum is a known phenomenon resulting from the aggregation of ejected atoms and atomic clusters, and presents a low-cost route for highly tunable nanoparticle synthesis, also in gas and liquid environments[11, 12, 13].

The in situ Raman spectra acquired before and after laser processing are shown in Figure 4e. The spectra have been normalized to the $\mathrm{E}_{2 g}^{1}$ peak and background-subtracted. Peak shifts indicative of different film thicknesses[14, 15] were not observed, suggesting that the thinning process was localized to the tip region of the flake, this region having been entirely removed by the time the post-ablation Raman spectrum was acquired. Energy-filtered TEM thickness mapping[8] of the flake showed that the remaining film was of uniform thickness, in support of the Raman results. In the post laser-processing TEM image of Figure $4 b$, an additional dark arc-shaped band is observed close the ablated region (marked with a green arrow), with additional smaller bands close by. Deformation of the pre-existing bands along the width of the flake (marked with yellow arrows) is also observed. Since we have ruled out mass-thickness contrast, we conclude that the dark bands observed are due to diffraction contrast, showing strain fields induced in the remaining $\mathrm{MoS}_{2}$ as a result of the ablation process. Strained mono-, bi-, and tri-layer $\mathrm{MoS}_{2}$ films are known to exhibit shifts in the $\mathrm{E}_{2 g}^{1}$ to $\mathrm{A}_{1 g}$ Raman modes and even splitting of the $\mathrm{E}_{2 g}^{1}$ mode for high strains[16, 17, 18, 19, 20]. However, as the number of layers increases, these strain effects are expected to become less pronounced.

Rather than peak shifts, our Raman results actually show an intensity change in the form of a decrease in the relative intensity of the $\mathrm{A}_{1 g}$ peak after laser processing. That is, the outof-plane $\mathrm{A}_{1 g}$ vibration became suppressed. It has been shown that the intensity of the $\mathrm{A}_{1 g}$ mode is very sensitive to electron doping, whereas the $\mathrm{E}_{2 g}^{1}$ mode is essentially unaffected by doping[21]. Hence we speculate that the suppression of the $\mathrm{A}_{1 g}$ peak may be due to crystal defects in the film resulting from photon irradiation. A confinement effect on the out-of-plane phonons of the $\mathrm{MoS}_{2}$ film due the nanocrystallites deposited on the film surface may also come into play, with a similar suppression of the $\mathrm{A}_{1 g}$ peak observed elsewhere for $\mathrm{MoS}_{2}$ trilayers decorated with $\mathrm{Au}$ nanoparticles[22]. However, we note that in addition to intensity changes, doped and nanoparticledecorated samples do in fact also exhibit their own subtle peak shifts, hence a thorough interpretation of the Raman spectra obtained here will rely on the results from further experiments where high-resolution TEM imaging may prove insightful.

\section{Conclusions}

In summary, the in situ assembly presented provides a means to perform laser-based processing of materials inside a TEM and to also probe the resulting microstructural response of the material by Raman spectroscopy, as demonstrated here in a laser-ablation experiment on $\mathrm{MoS}_{2}$ thin films. Complimentary analysis by electron-beam diffraction and EELS enable the generation of rich datasets all acquired in the same microscope. The experimental setup is thus highly versatile and could also be used for cathodoluminescence and photoluminescence studies.

The design approach presented here has the advantage of coupling the optical input and output signals directly to the sample chamber via vacuum feedthroughs (as opposed to passing through a window port on the microscope column). Thus power losses can be reduced and close approach of the probe/fiber to the specimen is enabled, which a) increases the solid angle for signal collection, and b) opens the door to near-field Raman probing with spot sizes smaller than those imposed by the 
diffraction limit, using an appropriate tapered optical fiber positioned a few nanometers from the specimen.

\section{Acknowledgments}

This work was performed at the Molecular Foundry, which is supported by the Office of Science, Office of Basic Energy Sciences, Scientific User Facilities Division, of the U.S. Department of Energy under Contract No. DE-AC02-05CH11231. The authors acknowledge funding from U.S. Department of Energy SBIR grant DE-FG02-07ER84813 awarded to Appliflex, LLC, and thank K. Schriver and H. Park for their support. We also thank A. King from Renishaw for support with the InVia Raman spectrometer, R. Forney from InPhotonics for support with the Raman probe, and S. Eyhusen from Zeiss for support with the installation of the in situ assembly on the Libra TEM.

\section{References}

[1] W. H. Weber, R. Merlin, Raman Scattering in Materials Science, Vol. 42, Springer Science \& Business Media, 2013.

[2] T. Dieing, O. Hollricher, J. Toporski, Confocal Raman Spectroscopy, Vol. 158, Springer Science \& Business Media, 2011.

[3] G. Wille, X. Bourrat, N. Maubec, A. Lahfid, Micron 67 (2014) 50.

[4] U. Schmidt, A. Jauss, W. Ibach, K. Weishaupt, O. Hollricher, Microscopy Today (2005) 30-34.

[5] Y. Ohno, Applied Physics Express 5 (2012) 125204.

[6] M. Picher, S. Mazzucco, S. Blankenship, R. Sharma, Ultramicroscopy 150 (2015) 10-15.

[7] B. Xiang, D. J. Hwang, J. B. In, S. G. Ryu, J. H. Yoo, O. Dubon, A. M. Minor, C. P. Grigoropoulos, Nano Letters 12 (2012) 2524-2529.

[8] R. F. Egerton, Electron Energy-Loss Spectroscopy in the Electron Microscope, 3rd Edition, Springer Science \& Business Media, 2011.

[9] T. J. Weitling, J. L. Verble, Physical Review B 3 (1971) 4286-4292.

[10] A. Castellanos-Gomez, M. Barkelid, A. M. Goossens, V. E. Calado, H. S. J. van der Zant, G. A. Steele, Nano Letters 12 (2012) 3187-3192.

[11] W. Marine, L. Patrone, B. Luk'yanchuk, M. Sentis, Applied Surface Science 154-155 (2000) 345-352.

[12] S. Amoruso, G. Ausanio, R. Bruzzese, M. Vitiello, X. Wang, Physical Review B 71 (2005) 033406.

[13] R. A. Ganeev, U. Chakravarty, P. A. Naik, H. Srivastava, C. Mukherjee, M. K. Tiwari, R. V. Nandedkar, P. D. Gupta, Applied Optics 46 (2007) 1205-1210.

[14] C. Lee, H. Yan, L. E. Brus, T. F. Heinz, J. Hone, S. Ryu, ACS Nano 5 (2010) 2695-2700.

[15] H. Li, Q. Zhang, C. C. R. Yap, B. K. Tay, T. H. T. Edwin, A. Olivier, D. Baillargeat, Advanced Functional Materials 22 (2012) 1385-1390.

[16] C. Rice, R. J. Young, R. Zan, U. Bangert, D. Wolverson, T. Georgiou, R. Jalil, K. S. Novoselov, Physical Review B 87 (2013) 081307(R).

[17] A. Castellanos-Gomez, R. Roldán, E. Cappelluti, M. Buscema, F. Guinea, H. S. J. van der Zant, G. A. Steele, Nano Letters 13 (2013) 5361-5366.

[18] H. J. Conley, B. Wang, J. I. Ziegler, R. F. H. Jr, S. T. Pantelides, K. I. Bolotin, Nano Letters 13 (2013) 3626-3630.

[19] Y. Y. Hui, X. Liu, W. Jie, N. Y. Chan, J. Hao, Y.-T. Hsu, L.-J. Li, W. Guo, S. Lau, ACS Nano 7 (2013) 7126-7131.

[20] C. R. Zhu, G. Wang, B. L. Liu, X. Marie, X. F. Qiao, X. Zhang, X. X. W. an H. Fan, P. H. Tan, T. Amand, B. Urbaszek, Physical Review B 88 (2013) 121301(R).

[21] B. Chakraborty, A. Bera, D. V. S. Muthu, S. Bhowmick, U. V. Waghmare, A. K. Sood, Physical Review B 85 (2012) 161403(R).

[22] T. S. Sreeprasad, P. Nguyen, N. Kim, V. Berry, Nano Letters 13 (2010) 4434-4441. 